\title{
CORRECTION
}

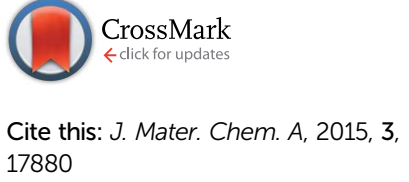

Cite this: J. Mater. Chem. A, 2015, 3, 17880

DOI: $10.1039 / c 5 \operatorname{ta} 90169 c$

www.rsc.org/MaterialsA

\section{Correction: Ultrafast high-performance extraction of uranium from seawater without pretreatment using an acylamide- and carboxyl-functionalized metal-organic framework}

Ling Ling Wang, Feng Luo, ${ }^{*}$ Li Long Dang, Jian Qiang Li, Xiao Liu Wu, Shu Juan Liu and Ming Biao Luo

Correction for 'Ultrafast high-performance extraction of uranium from seawater without pretreatment using an acylamide- and carboxyl-functionalized metal-organic framework' by Ling Ling Wang et al., J. Mater. Chem. A, 2015, 3, 13724-13730.

The experimental section was omitted from the above manuscript and is now available as ESI, downloadable from the article web page.

The Royal Society of Chemistry apologises for these errors and any consequent inconvenience to authors and readers. 